\title{
Burrowing behavior as an indicator of post-laparotomy pain in mice
}

\author{
Paulin Jirkof ${ }^{1}$, Nikola Cesarovic $^{1}$, Andreas Rettich' ${ }^{1}$, Flora Nicholls ${ }^{2}$, Burkhardt Seifert ${ }^{3}$ and Margarete Arras ${ }^{1,4}$ * \\ Institute of Laboratory Animal Science, University of Zurich, Zurich, Switzerland \\ 2 Central Biological Laboratory, University Hospital Zurich, Switzerland \\ ${ }^{3}$ Biostatistics Unit, Institute of Social and Preventive Medicine, University of Zurich, Zurich, Switzerland \\ ${ }^{4}$ Division of Surgical Research, University Hospital Zurich, Zurich, Switzerland
}

\section{Edited by:}

Carmen Sandi, Ecole Polytechnique

Federale De Lausanne, Switzerland

\section{Reviewed by:}

Guillaume Poirier, Ecole Polytechnique Fédérale De Lausanne, Switzerland Patrizia Campolongo, Università degli Studi di Roma La Sapienza, Italy

\section{*Correspondence:}

Margarete Arras, Division of Surgical Research, University Hospital Zurich, Sternwartstrasse 6, CH-8091 Zurich, Switzerland.

e-mail: arras@ltk.uzh.ch
Detection of persistent pain of a mild-to-moderate degree in laboratory mice is difficult because mice do not show unambiguous symptoms of pain or suffering using standard methods of shortterm observational or clinical monitoring. This study investigated the potential use of burrowing performance - a spontaneous and highly motivated behavior - as a measure of post-operative pain in laboratory mice. The influence of minor surgery on burrowing was investigated in adult C57BL/6J mice of both genders in a modified rodent burrowing test (displacement of food pellets from a pellet-filled tube) within the animal's home cage. Almost all (98\%) healthy mice burrowed (mean latency $1.3 \mathrm{~h}, \mathrm{SEM} 0.5 \mathrm{~h}$ ). After surgery without pain treatment, latency of burrowing was significantly prolonged (mean $\Delta$ latency $10 \mathrm{~h}$ ). Analgesic treatment using the anti-inflammatory drug carprofen ( $5 \mathrm{mg} / \mathrm{kg}$ bodyweight) decreased latency of burrowing after surgery (mean $\Delta$ latency $5.5 \mathrm{~h}$ ) to the level found in mice that had been anesthetized (mean $\Delta$ latency $5.4 \mathrm{~h}$ ) or had received anesthesia and analgesia (mean $\Delta$ latency $4.6 \mathrm{~h}$ ). Analgesia during surgery was associated with a significantly earlier onset of burrowing compared to surgery without pain treatment. A distinct gradation in burrowing performance was found ranging from the undisturbed pre-operative status to the intermediate level following anesthesia/analgesia and surgery with analgesia, to the pronounced prolongation of latency to burrow after surgery without pain relief. In conclusion, post-surgical impairment of general condition, probably mainly attributable to pain, can be conveniently assessed in laboratory mice on the basis of the burrowing test.

Keywords: post-operative pain, pain assessment, C57BL/6J mice, burrowing, species-typical behavior, analgesia, post-laparotomy pain, post-surgical pain

\section{INTRODUCTION}

The detection of pain in laboratory animals is essential for both scientific and ethical reasons. The responsibility to ensure the care and welfare of the millions of animals used annually in biomedical research (Baumans, 2004, 2005) requires the recognition and alleviation of pain caused by animal experiments (Gauthier and Griffin, 2005). Furthermore, methods of gauging pain are needed in basic research focused on the mechanisms and patho-physiology of pain, and in the applied context of searching for new and improved painkillers. Such biomedical research is often conducted in mice currently the most widely used laboratory animal species due to the availability of various genetically modified mice models (Baumans, 2004, 2005). However, diagnosis of pain in the mouse is challenging because this species does not voluntarily exhibit obvious signs of pain, which can be explained by the fact that, as prey animals, mice try to hide signs of pain, suffering or disability in order to avoid attracting predators (Stasiak et al., 2003; Peterson, 2004; van Sluyters and Obernier, 2004).

Extensive research in this field has led to a multitude of analgesiometric tests and models for the detection of acute pain and the identification of allodynia or hyperalgesia in mice (e.g., tail flick latency test, paw licking response, partial sciatic nerve injury model;
Chapman et al., 1985; Le Bars et al., 2001; Mogil, 2009). Nevertheless, the assessment of non-acute or persistent pain, for example from surgery or other tissue trauma, which can last a few hours to days, remains difficult in the mouse (Kohn et al., 2007). Signs and symptoms of prolonged pain stages published in articles and guidelines usually refer to severe pain due to major surgery, highly invasive, or otherwise severely noxious interventions. Obvious symptoms such as sunken flanks, neglected grooming, piloerection, hunched back or immobility are clear evidence of a severely impaired, often moribund, health status in mice. Interventions with a lesser impact seem not to evoke such clearly recognizable changes. For example, after routine surgical interventions like laparotomy, mice are well known not to display any easily observable abnormal appearance, posture, or behavior indicating the presence of pain, thus leading to a demand for the development of standard evaluation methods for mild-to-moderate pain (Hawkins, 2002). As behavior can be observed easily in a non-invasive manner and can provide meaningful cues as to the internal state of an animal, analyzing complex behavioral changes (e.g., nest building behavior) in pain states has frequently been suggested as a promising approach to assess both pain severity and the efficiency of pain management regimes (Jourdan et al., 2001; Mogil and Crager, 2004; Mogil, 2009; 
Roughan et al., 2009). Currently, examples of extensive and refined ethological measures in mice after laparotomy in their home cage environment have been introduced. Global changes in the frequency of spontaneous behaviors (e.g., rearing, sniffing, walking; Roughan et al., 2009), and in the quality of nest construction and structuring of territory (home cage/areal; Arras et al., 2007) under post-operative pain have been described. However, while the former method is based on specialized automated software analysis, the latter has not yet been standardized, which hampers translation of both methods to a laboratory routine setting.

A promising and simple experimental setup with which to assess changes in spontaneous burrowing behavior correlating with different impairments in rodents was published by Deacon and coauthors (Deacon et al., 2001; Deacon, 2006). The test is based on the species-typical behavior of mice to spontaneously displace items from tubes within their home cage; this behavior was used as a tool to provoke and determine burrowing behavior under experimental conditions in models of prion disease (Deacon et al., 2001), brain lesions (Deacon et al., 2003), systemic inflammation (Teeling et al., 2007), and Alzheimer's disease (Deacon et al., 2008). In these models, a decrease in burrowing behavior was interpreted to be correlated with chronic neurological or immunological disorders. As burrowing behavior, although highly motivated (Sherwin et al., 2004), is not an essential maintenance behavior like food consumption or sleeping, we suggest that it is sensitive to different, subtle kinds of impact on the animals' general condition and wellbeing. Changes in this species-typical behavior might therefore be useful as an indicator of pain after surgery.

We used a modified version of the burrowing test setting within each animal's home cage to determine the pre- and post-operative burrowing performance of male and female mice. To induce postoperative pain we performed a sham vasectomy or embryo transfer, both including a laparotomy (Martin et al., 2004; Arras et al., 2007; Roughan et al., 2009). To distinguish between the effects of surgery, anesthesia, and analgesic treatment, we tested groups of mice that underwent surgery with or without the analgesic carprofen or procedural control treatments with anesthesia only or anesthesia plus analgesia. The results presented establish the reliability and feasibility of an easy-to-perform burrowing test with the aim of assessing post-operative pain in mice.

\section{MATERIALS AND METHODS}

The animal housing and experimental protocols were approved by the Cantonal Veterinary Department, Zurich, Switzerland, under license no. ZH 120/2008, and were in accordance with Swiss Animal Protection Law. Housing and experimental procedures also conform to the European Convention for the Protection of Vertebrate Animals used for Experimental and other Scientific Purposes (Council of Europe nr. 123 Strasbourg 1985) and to the Guide for the Care and Use of Laboratory Animals (Institute of Laboratory Animal Resources, National Research Council, National Academy of Sciences, 1996).

\section{ANIMALS}

The animals were 32 male and 32 female C57BL/6J mice obtained from our in-house breeding facility at the age of 6-8 weeks. All animals were housed in groups of three to eight animals of the same sex for at least 3 weeks prior to testing in our animal room. Animals were kept in type 3 clear-transparent plastic cages $(425 \mathrm{~mm} \times 266 \mathrm{~mm} \times 155 \mathrm{~mm})$ with autoclaved dust-free sawdust bedding and two nestlets ${ }^{\mathrm{TM}}$ (each $5 \mathrm{~cm} \times 5 \mathrm{~cm}$ ), consisting of cotton fibers (Indulab AG, Gams, Switzerland) as nesting material. They were fed a pelleted and extruded mouse diet (Kliba No. 3436, Provimi Kliba, Kaiseraugst, Switzerland) ad libitum (provided in the food hopper continuously throughout the entire duration of the experiment) and had unrestricted access to sterilized drinking water. The light/dark cycle in the room consisted of 12/12 h (lights on 15:00, lights off 03:00) with artificial light (40 Lux in the cage). The temperature was $21 \pm 1^{\circ} \mathrm{C}$, with a relative humidity of $50 \pm 5 \%$, and the air pressure was controlled at $50 \mathrm{~Pa}$ with 15 complete changes of filtered air per hour (HEPA H 14 filter). The animal room was insulated to prevent electronic and other noise. Disturbances, e.g., visitors or unrelated experimental procedures, were not allowed.

\section{EXPERIMENTAL SETUP}

As a burrowing apparatus, a plastic bottle (standard opaque water bottle, $250 \mathrm{ml}, 150 \mathrm{~mm}$ length, $55 \mathrm{~mm}$ diameter) filled with 138 $142 \mathrm{~g}$ of food pellets identical to those of the animal's normal diet was used. An additional empty bottle of the same dimensions was provided to serve as a shelter for the animal (Figure 1).

\section{EXPERIMENTAL DESIGN}

To test the effects of surgery on burrowing behavior, each animal was tested in the burrowing apparatus before (baseline) and after an experimental procedure (experimental). The individual baseline values were used to compensate for inter-individual variation in burrowing behavior. Eight animals of each sex were allocated randomly to one of four treatment groups: (1) the "surgery + anesthesia" group, which underwent anesthesia and minor surgery without analgesic treatment; (2) the "surgery + anesthesia + analgesia" group, which underwent anesthesia and a minor surgery with analgesic treatment, (3) the "anesthesia" group, or (4) the "anesthesia + analgesia" group; groups 3 and 4 being procedural control groups that underwent only anesthesia with (4) or without (3) analgesia (Figure 2).

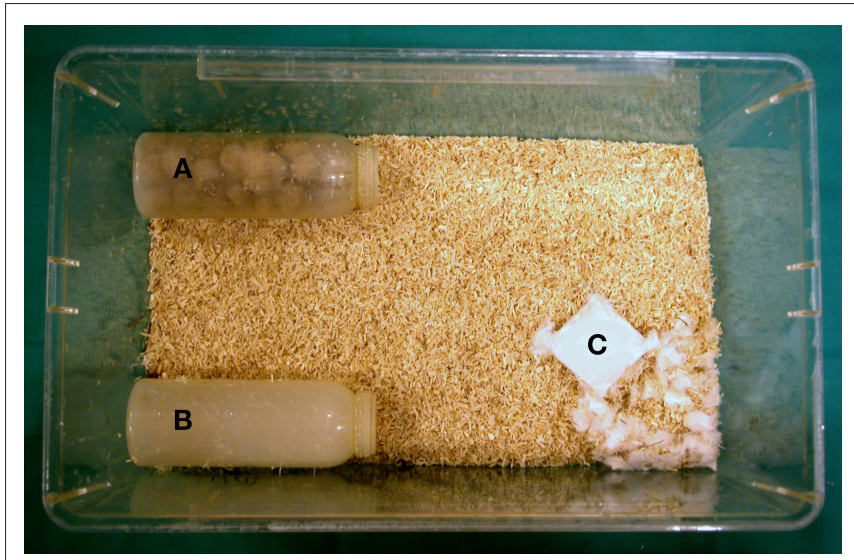

FIGURE 1 | Experimental setup. Burrowing test apparatus (A), shelter (B), and nesting material $(\mathbf{C})$ in home cage. 


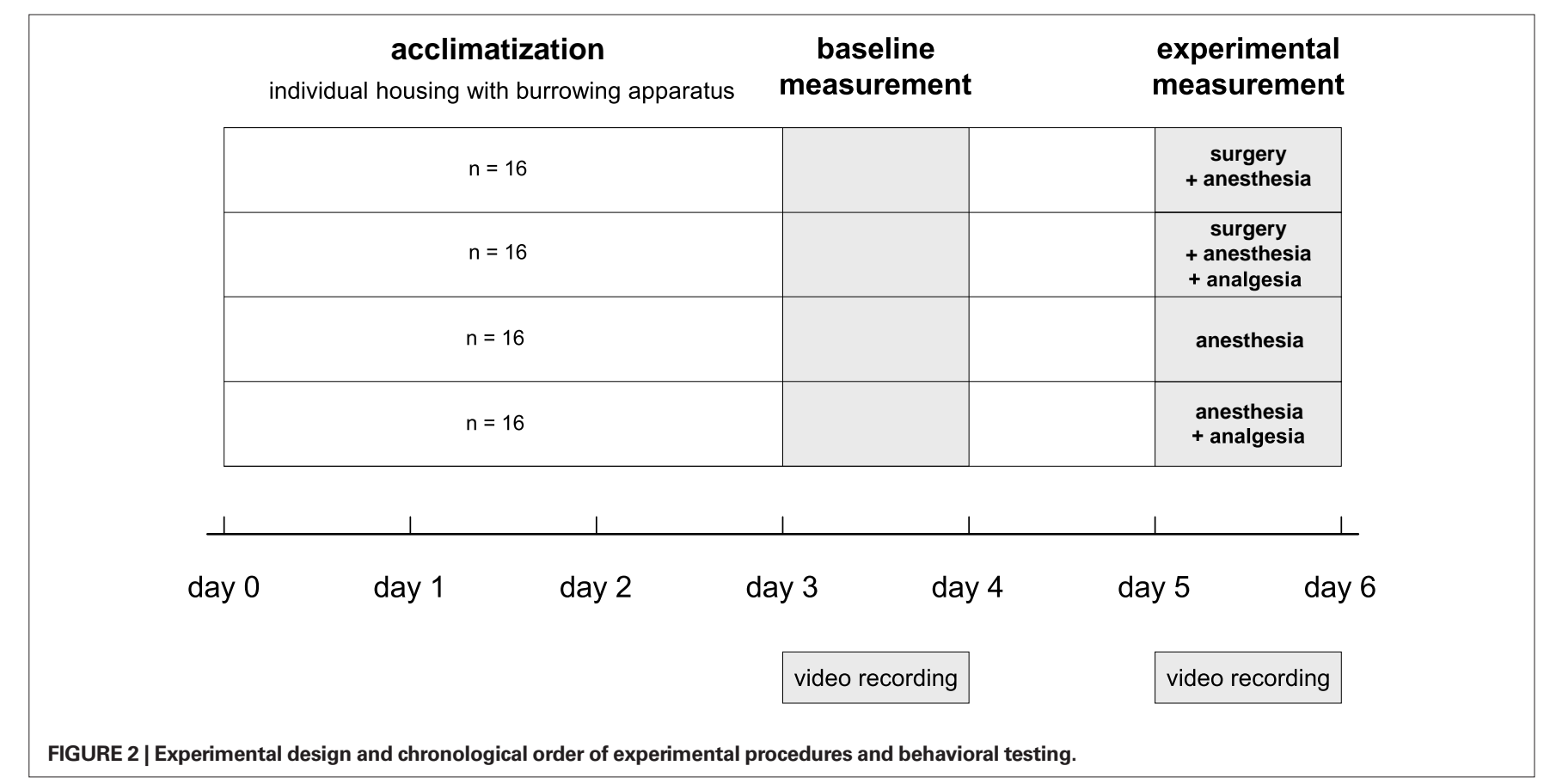

\section{ACCLIMATIZATION AND BASELINE MEASUREMENTS}

For acclimatization, animals were housed individually for 3 days in a standard cage with food and water ad libitum containing the experimental setup as described above (Figure 1).

Other than this prior experience with the burrowing apparatus, the animals had no experience with behavioral testing and were not selected for burrowing performance.

Measurements of baseline values of burrowing behavior started at 15:00 hours and were carried out in the animal room. Food pellets removed or eaten by the mice during the preceding days were replaced in the apparatus before testing started. All tests were recorded digitally for $24 \mathrm{~h}$ in the absence of a human observer with an infrared-sensitive camera fixed above the cage.

\section{TREATMENTS AND EXPERIMENTAL MEASUREMENTS}

Experimental measurements of burrowing behavior were performed 2 days after baseline measurements. The treatment began at 13:00 hours with a subcutaneous injection of $2 \mu \mathrm{l} / \mathrm{g}$ body weight of phosphate buffered saline (PBS) for the surgery + anesthesia and anesthesia groups. In the surgery + anesthesia + analgesia and anesthesia + analgesia groups, $5 \mathrm{mg} / \mathrm{kg}$ body weight of the analgesic carprofen (Rimadyl ${ }^{\mathrm{TM}}$, Pfizer Inc., NY, USA) was diluted in PBS and injected as $2 \mu \mathrm{l} / \mathrm{g}$ body weight. Forty-five minutes later, the animals were transferred in individual transport cages to the operating theater, which was located nearby. Mice were anesthetized with sevoflurane (Sevorane ${ }^{\mathrm{TM}}$, Abbott, Baar, Switzerland) as a mono-anesthesia. The anesthetic gas was provided with a rodent inhalation anesthesia apparatus (Provet, Lyssach, Switzerland); pressurized air was used as carrier gas. After induction of anesthesia in a Perspex induction chamber $(8 \%$ sevoflurane, $600 \mathrm{ml} / \mathrm{min}$ airflow) animals were transferred to a warming mat (Gaymar, TP500, Orchard Park, NY, USA) set at $39^{\circ} \pm 1^{\circ} \mathrm{C}$ to ensure constant body temperature and anesthesia was maintained via nose mask (4.9\% sevoflurane, $600 \mathrm{ml} / \mathrm{min}$ airflow). The fur was clipped and the operating field disinfected with ethanol in all animals. Male and female mice of both surgery groups underwent a one-side sham vasectomy or a one-side sham embryo transfer, respectively. The incision in the abdominal muscle wall was closed with absorbable sutures (Vicryl ${ }^{\mathrm{TM}}$, 6/0 polyglactin 910, Ethicon Ltd, Norderstedt, Germany) and the skin was closed using skin staples (Precise ${ }^{\mathrm{TM}}$, $3 \mathrm{M}$ Health Care, St Paul, MN, USA). Surgery was completed within 6-8 $\mathrm{min}$ in both surgery groups. Anesthesia lasted $14-16 \mathrm{~min}$ in all four treatment groups. Animals were allowed to recover for 15-20 min on the warming mat before they were transferred back to the animal room for subsequent behavioral testing.

The burrowing test began at 15:00 hours by returning the mouse from its transport cage to its home cage containing the refilled test apparatus and by starting the digital video recording.

\section{BEHAVIORAL ANALYSIS}

The recorded 24-h video sequences were analyzed using ObserverXT ${ }^{\mathrm{TM}}$ software (Noldus, Wageningen, Netherlands). Burrowing was defined as the removal of more than three pellets from the apparatus within $10 \mathrm{~s}$. The latency to onset of burrowing behavior (latency to burrow), and the duration between the start of burrowing and the total emptying of the apparatus (duration of burrowing), were measured in seconds. Additionally, to allow comparison with existing literature, the weight (in grams) of food pellets removed after $2 \mathrm{~h}$ of testing was calculated indirectly by counting pellets removed on video files.

\section{CLINICAL INVESTIGATION}

Animals were investigated for abnormalities of appearance (e.g., fur, eyes), posture, and movement at the end of the baseline and experimental recordings (15:00 hours at day 4 and day 6) according to a routinely used scoring system (Arras et al., 2007). 


\section{STATISTICAL ANALYSIS}

All statistical analyses were performed with SPSS 17.0 software (SPSS Inc., Chicago, IL, USA).

Mean and standard error of the mean (SEM) of latency to burrow, duration of burrowing and weight of removed food pellets were calculated for the baseline measurements and for experimental measurements of the four treatment groups. Additionally, mean, SEM and the 95\% confidence interval were calculated for the baseline latency to burrow and the duration of burrowing of all 64 animals.

To consider inter-individual variation the individual baseline values were used to compare experimental latencies, durations and pellet weights between groups. Therefore, mean and SEM were additionally calculated for all groups using the differences $(\Delta)$ in latencies, durations and pellet weights between experimental and baseline measurements of each animal.

All data was tested for normal distribution and homogeneity of variance. With the exception of the weight of removed food pellets, all data met the necessary assumptions for parametric analyses.

Absolute and $\Delta$ latencies and durations were compared between genders with independent two-sample $t$-tests, with absolute and $\Delta$ weight of removed pellets being compared with the Mann-Whitney $U$ test.

Dependent $t$-tests for paired samples were used for the comparison of baseline and experimental absolute latencies and durations of each experimental group. To compare baseline and experimental values of weights of removed pellets of each group, the Wilcoxon test for paired data was used.

Experimental absolute and $\Delta$ latencies and durations between groups were compared using a one way analysis of variance (ANOVA); post hoc testing was conducted with the Tukey test. To compare absolute and $\Delta$ weights of removed pellets between groups, the Kruskal-Wallis test was performed.
Kaplan-Meier survival analysis to examine the distribution of time to effect (latency to burrow) for the four different treatments was performed. To test whether the latency to burrow differed statistically between treatment groups, a log rank significance test was performed.

Significance for all statistical tests was established at $p<0.05$.

\section{RESULTS}

No significant effect of the animals' gender was detected with any of the measures. Therefore results are reported for the combined data set of males and females.

\section{BURROWING PERFORMANCE}

Figure 3 presents the percentage change in the fraction of mice that started burrowing during baseline and experimental recordings. Out of 64 animals only one male did not burrow, either during the $24 \mathrm{~h}$ of baseline recording or during the $24 \mathrm{~h}$ of experimental recording. During the $24 \mathrm{~h}$ baseline recordings, the majority (89\%) of mice started burrowing within the first hour of the test, whereas most mice in all the experimental groups started burrowing considerably later. The fraction of mice that started burrowing increased similarly in the anesthesia, anesthesia + analgesia and surgery + anesthesia + analgesia groups within $12 \mathrm{~h}$ of observation. While anesthesia and surgery + analgesia groups reached baseline values after 13 and $16 \mathrm{~h}$ of recording, respectively, the anesthesia + analgesia group did not reach baseline values until $23 \mathrm{~h}$. The fraction of mice starting burrowing in the surgery + anesthesia group was distinctly lower and did not reach values of baseline recordings during the $24 \mathrm{~h}$ of observation.

\section{BASELINE VS. EXPERIMENTAL VALUES}

During baseline measurements, mice started burrowing on average at $1.3 \mathrm{~h}$, SEM $0.5 \mathrm{~h}$ (95\% confidence interval: 0.32 , $2.3 \mathrm{~h}$ ) and emptied the burrowing apparatus within $0.83 \mathrm{~h}, \mathrm{SEM}$

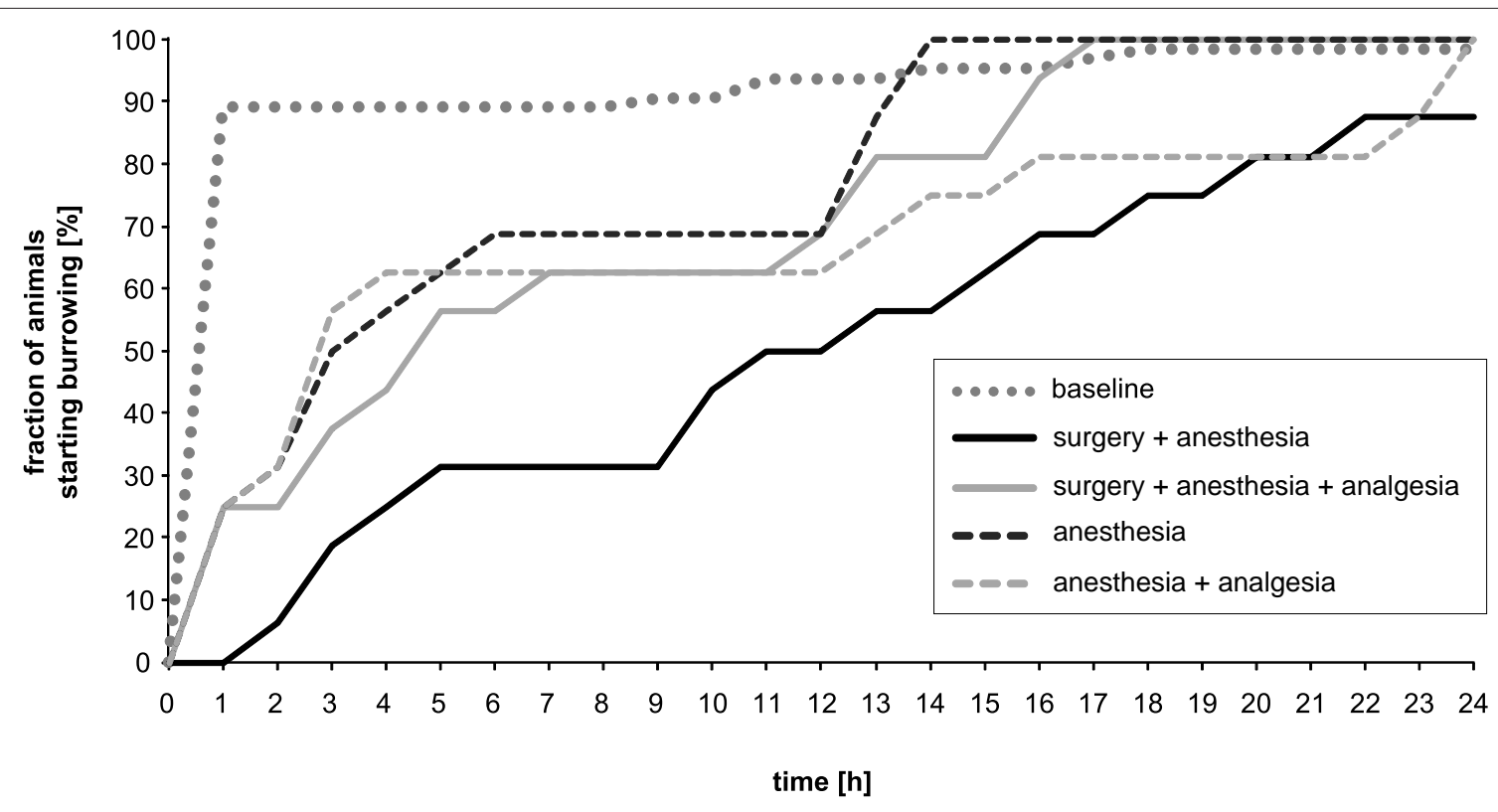

FIGURE 3 | Percentage of mice/hour that started burrowing at baseline vs. experimental conditions. Healthy mice started burrowing immediately, mostly within $1 \mathrm{~h}$ (baseline). Between-treatment differences were greatest between hours 7 and 9 . 
$0.33 \mathrm{~h}$ (95\% confidence interval: $0.17,1.5 \mathrm{~h})$. All the experimental treatments caused marked changes in the latency to burrow, duration of burrowing and the weight of removed pellets at $2 \mathrm{~h}$.

Compared to the respective baseline values, mice showed significantly longer latencies after experimental treatment in the surgery + anesthesia group, surgery + anesthesia + analgesia group, and anesthesia group, but a tendency toward longer experimental latencies only in the anesthesia + analgesia group (Table $\mathbf{1}$ ).

Mean of duration of burrowing tended to be higher after all experimental treatments compared to baseline values, but this difference could not be confirmed as statistically significant (Table 1).

The amount of pellets removed at $2 \mathrm{~h}$ after treatment was significantly less than baseline values in all four groups (Table 1).

\section{EFFECTS OF TREATMENT ON BURROWING BEHAVIOR}

Comparison of experimental groups revealed distinct differences in the effects of the respective treatments on latency to burrow and amount of pellets removed at $2 \mathrm{~h}$. Surgery without pain relief caused considerably longer absolute and $\Delta$ latencies and lower weight of removed pellets than surgery with analgesia, anesthesia, or anesthesia + analgesia but no relevant difference in absolute and $\Delta$ duration of burrowing (for detailed results see Tables 1 and 2).

No statistically significant difference could be observed either in absolute latency to burrow, duration of burrowing and weights of pellets, $\Delta$ latency and $\Delta$ duration, while $\Delta$ amounts of removed pellets show significant differences (Table 3 ).

The distribution of time to effect, i.e., latency to burrow traced in Kaplan-Meier analysis, exhibited a distinct shift of the surgery+ anesthesia group compared to the other three treatment groups (Figure 4). The log rank analysis showed a significant association between treatment of groups and onset of burrowing: the surgery+ anesthesia group started to burrow significantly later compared to the surgery + anesthesia + analgesia group and the anesthesia group. All other group comparisons showed no significant differences (surgery + anesthesia + analgesia vs. anesthesia: $p=0.260, \chi^{2}=1.270$; surgery + anesthesia + analgesia vs. anesthesia + analgesia: $p=0.479, \chi^{2}=0.502$; anesthesia vs. anesthesia + analgesia: $\left.p=0.180, \chi^{2}=1.798\right)$.

\section{CLINICAL INVESTIGATION}

Observations of appearance, posture, and movement revealed no difference between baseline and experimental measures, or between experimental groups.

Table 1 | Absolute values of latency to burrow, duration of burrowing and weight of removed food pellets. Results of paired t-test for latency to burrow and duration of burrowing, and Wilcoxon test for removed pellet weights.

\begin{tabular}{|c|c|c|c|c|c|c|}
\hline & & & Mean (h) & SEM (h) & $p$-Value & $t$ \\
\hline \multirow[t]{8}{*}{ Latency to burrow } & Surgery + anesthesia & Baseline & 0.09 & 0.02 & $0.0005^{*}$ & -5.779 \\
\hline & & Experimental & 10.41 & 1.79 & & \\
\hline & Surgery + anesthesia + analgesia & Baseline & 1.58 & 1.02 & $0.004^{*}$ & -3.431 \\
\hline & & Experimental & 7.09 & 1.66 & & \\
\hline & Anesthesia & Baseline & 0.10 & 0.04 & $0.001^{*}$ & -4.07 \\
\hline & & Experimental & 5.48 & 1.32 & & \\
\hline & Anesthesia + analgesia & Baseline & 3.38 & 0.49 & $0.081^{\text {n.s. }}$ & -1.871 \\
\hline & & Experimental & 7.90 & 2.16 & & \\
\hline \multirow[t]{9}{*}{ Duration of burrowing } & Surgery + anesthesia & Baseline & 0.27 & 0.01 & $0.193^{\text {n.s. }}$ & -1.387 \\
\hline & & Experimental & 1.89 & 0.27 & & \\
\hline & Surgery + anesthesia + analgesia & Baseline & 0.46 & 0.31 & $0.148^{\text {n.s. }}$ & 1.601 \\
\hline & & Experimental & 1.63 & 0.38 & & \\
\hline & Anesthesia & Baseline & 0.45 & 0.13 & $0.190^{\text {n.s. }}$ & -1.377 \\
\hline & & Experimental & 1.85 & 0.23 & & \\
\hline & Anesthesia + analgesia & Baseline & 0.66 & 0.08 & $0.194^{\text {n.s. }}$ & -1.405 \\
\hline & & Experimental & 3.81 & 0.37 & & \\
\hline & & & Mean (g) & $\operatorname{SEM}(g)$ & $p$-Value & Z-value \\
\hline \multirow{8}{*}{$\begin{array}{l}\text { Weight of removed food } \\
\text { pellets at } 2 \mathrm{~h}\end{array}$} & Surgery + anesthesia & Baseline & 134.1 & 5.9 & $0.0005^{*}$ & -3.556 \\
\hline & & Experimental & 10.3 & 9.3 & & \\
\hline & Surgery + anesthesia + analgesia & Baseline & 134.3 & 5.7 & $0.001^{*}$ & -3.357 \\
\hline & & Experimental & 35 & 15.7 & & \\
\hline & Anesthesia & Baseline & 133.2 & 5.2 & $0.001^{*}$ & -3.438 \\
\hline & & Experimental & 30.4 & 13.8 & & \\
\hline & Anesthesia + analgesia & Baseline & 86.2 & 15.4 & $0.004^{*}$ & -2.852 \\
\hline & & Experimental & 33 & 14.1 & & \\
\hline
\end{tabular}

${ }^{*}=p<0.05 ;$ n.s. $=p>0.05$ 
Table 2 | Delta $(\Delta)$ values of latency to burrow, duration of burrowing, and weight of removed food pellets.

\begin{tabular}{|c|c|c|c|}
\hline & & Mean (h) & SEM (h) \\
\hline$\Delta$ Latency & Surgery + anesthesia & 10.3 & 1.8 \\
\hline \multirow[t]{3}{*}{ to burrow } & Surgery + anesthesia + analgesia & 5.5 & 1.6 \\
\hline & Anesthesia & 5.4 & 1.3 \\
\hline & Anesthesia + analgesia & 4.6 & 1.6 \\
\hline$\Delta$ Duration & Surgery + anesthesia & 1.6 & 0.2 \\
\hline \multirow[t]{4}{*}{ of burrowing } & Surgery + anesthesia + analgesia & 1.2 & 0.5 \\
\hline & Anesthesia & 1.4 & 0.2 \\
\hline & Anesthesia + analgesia & 3.3 & 0.5 \\
\hline & & Mean (g) & SEM $(g)$ \\
\hline$\Delta$ Weight of & Surgery + anesthesia & -123.7 & 10.6 \\
\hline removed food & Surgery + anesthesia + analgesia & -99.3 & 15.8 \\
\hline \multirow[t]{2}{*}{ pellets at $2 \mathrm{~h}$} & Anesthesia & -102.8 & 13.8 \\
\hline & Anesthesia + analgesia & -53.4 & 16.0 \\
\hline
\end{tabular}

Table 3 | Results of ANOVA for experimental absolute and $\Delta$ latencies to burrow and durations of burrowing and of Kruskall-Wallis test for experimental absolute and $\Delta$ weights of removed food pellets for all four groups.

\begin{tabular}{lll}
\hline Group comparison & $\boldsymbol{p}$-Value & F-value \\
\hline Absolute latency to burrow & $0.283^{\text {n.s. }}$ & 1.301 \\
Absolute duration of burrowing & $0.530^{\text {n.s. }}$ & 0.746 \\
$\Delta$ Latency to burrow & $0.139^{\text {n.s. }}$ & 1.906 \\
$\Delta$ Duration of burrowing & $0.222^{\text {n.s. }}$ & 1.518 \\
\hline & $\boldsymbol{p}$-Value & $\boldsymbol{\chi}^{\mathbf{2}}$ \\
\hline Weight of removed food pellets at $2 \mathrm{~h}$ & $0.693^{\text {n.s. }}$ & 1.452 \\
$\Delta$ Weight of removed food pellets at $2 \mathrm{~h}$ & $0.005^{*}$ & 12.791 \\
\hline
\end{tabular}

${ }^{*}=p<0.05 ;$ n.s. $=p>0.05$.

\section{DISCUSSION}

The burrowing test (modified from descriptions by Deacon et al., 2001), was investigated for its feasible use in the assessment of post-operative general condition and probably post-operative pain. The test proved easy to perform in an experimental setting within the animal's home cage.

All burrowing parameters analyzed responded sensitively to the different experimental manipulations performed on the animals. Healthy mice of both genders of the common C57BL/6J strain started burrowing (i.e., displaced substantial numbers of food pellets from a tube) in general within $0.3-2.3 \mathrm{~h}$ after this pellet-filled tube was provided to them. After surgery without pain treatment, the latency to burrow was significantly prolonged, i.e., when the pellet-filled tube was presented to them in their home cage after surgery mice started burrowing several hours later. With analgesic treatment, the latency to burrow after surgery was only moderately extended to a level similar to that found in the procedural control groups, i.e., in mice anesthetized only or in mice receiving anesthesia and pain treatment only. Thus, surgery with analgesic treatment in mice resulted in a significantly earlier onset of burrowing compared to surgery without pain relief.

Likewise, the weight of removed pellet material in the default time frame of $2 \mathrm{~h}$ (referred to by Deacon et al., 2001) was significantly reduced after any of the treatments. Comparing between treatments, the amount of removed pellets was less after surgery than after surgery with analgesia, anesthesia and analgesia, or anesthesia only. The duration of burrowing (i.e., the time required by the animal to empty the tube) was not significantly different between any of the groups although it was prolonged after all experimental treatments compared to the healthy baseline status.

In our laparotomy model, the results of the assessment of burrowing performance were as expected from common sense, as well as from earlier studies on physiology (Arras et al., 2007), pain research (Langford et al., 2010), and from the results of previously published burrowing testing in different kinds of disorders, including pathological processes in the brain such as prion disease, pre-frontal cortex or hippocampal lesions (Deacon et al., 2001, 2002, 2003, 2008; Teeling et al., 2007). Such brain damage can alter measures of burrowing independently of pain. Burrowing, as a spontaneous behavior, can also be influenced by other factors not directly related to pain such as motivation (Sherwin et al., 2004), fear, anxiety, distress, suffering, olfactory irritants and aversive situations, or generally by depressed general condition and changes in overall wellbeing. Therefore, control experiments were conducted to distinguish between the impact of anesthesia and analgesia per se and of surgery as the pain-inducing insult. To estimate the impact of pain, surgery was conducted either with or without pain relief. Moreover, care was taken to prevent any other surrounding factors unrelated to pain from influencing the experiments, i.e., the animal room was insulated and animals were not disturbed by the presence of any personnel in the post-operative time frame in which video recording was performed and burrowing took place.

The burrowing setting described previously by Deacon was adapted in our approach as we used smaller tubes and carried out the test at a different time of day. These modifications had negligible influence, since we had found in a preliminary experiment in our laboratory (data not shown) that burrowing performance, especially the latency to burrow, was largely independent of circadian rhythm, i.e., the time of day of testing. Animals were allowed to habituate to the test apparatus during the acclimatization period since Deacon showed that healthy mice increased burrowing activity at the second trial and then continued their high level of burrowing (Deacon et al., 2001). In another, not yet published, study using this test repeatedly, we found only slight oscillations of baseline burrowing performance, but no tendency toward longer or shorter latencies to burrow or burrowing duration (data not shown). To exclude disturbances that will induce fear, agitation, or any kind of distress, and thus certainly have impact on the animals' spontaneous behavior, we avoided any human presence in the critical time frame, and no-one handled cages or animals during this time. Hence, for the appraisal, the pellets were not weighed at a predetermined time point (i.e., after $2 \mathrm{~h}$; Deacon et al., 2001), but rather the weight of the removed pellets was calculated by counting them from video recordings at a certain time point. This procedure gave results comparable to those known from 


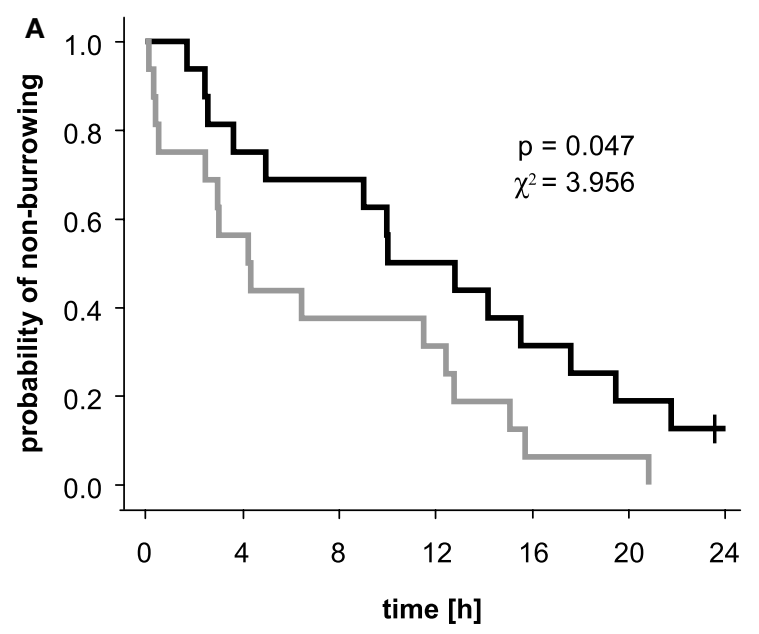

surgery + anesthesia

surgery + anesthesia + analgesia

C

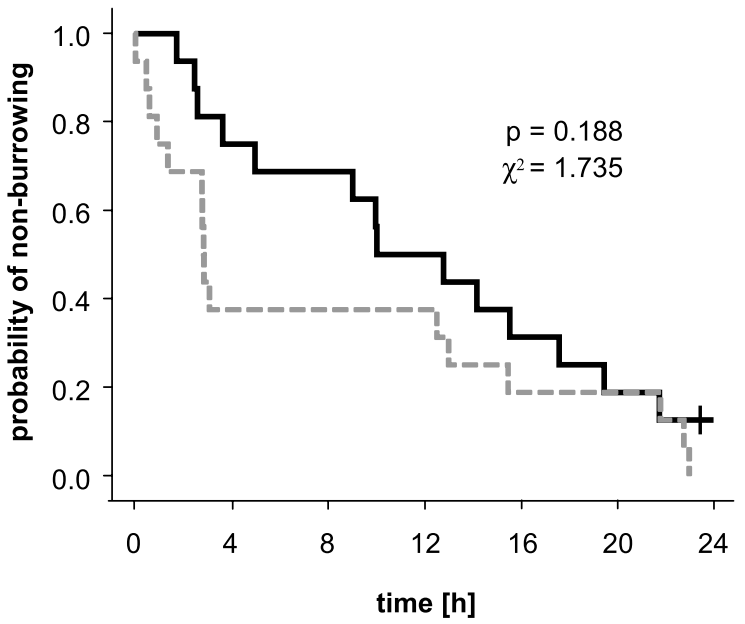

surgery + anesthesia

anesthesia + analgesia
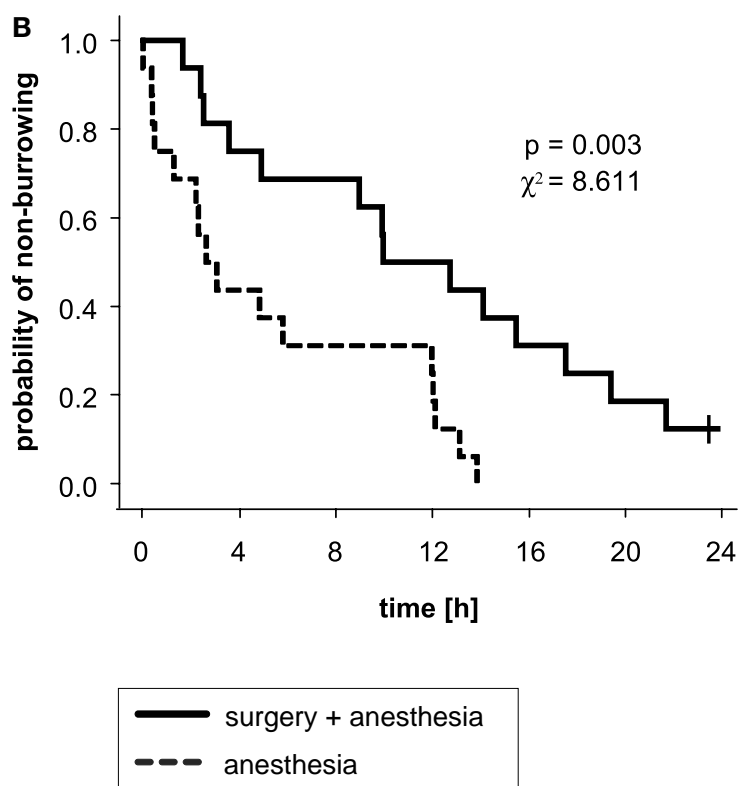

D

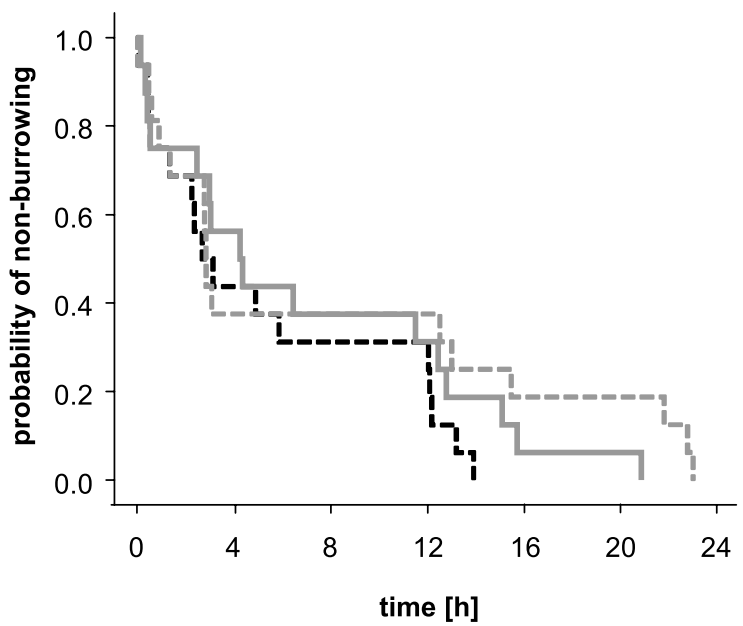

_ surgery +anesthesia +analgesia
- anesthesia
- anesthesia +analgesia

and surgery without pain relief and mice that were anesthetized only. (C) The difference between the surgery + anesthesia group and the anesthesia + analgesia group was not statistically significant. (D) No significant differences in latencies to burrow were found between surgery + anesthesia + analgesia, anesthesia and anesthesia + analgesia groups. the literature (Deacon et al., 2001). Thus, with the advantage of video recording, we could follow the activities of each mouse in its home cage without disturbing the animal by handling the tube.
The infrared-sensitive video recording allowed us to determine the latency to burrow accurately, and to define the time frame over which differences in burrowing performance were greatest between 
groups receiving different treatments. In this way we could show that less than $30 \%$ of mice that had undergone surgery without pain alleviation had started burrowing 7-9 h post-operatively. In contrast, most animals in which a pain killer was administered during surgery $(62 \%)$, as well as those mice that received only anesthesia $(70 \%)$ or anesthesia and analgesia (62\%), had started burrowing by $7-9 \mathrm{~h}$ after surgery.

As the construction of burrows offers advantages as protection from natural threats (e.g., predation, detrimental environmental conditions) in the wild, burrowing is highly motivated behavior, and persists as spontaneous behavior during domestication in the laboratory mouse (Adams and Boice, 1981; Dudek et al., 1983; Sherwin et al., 2004). As burrowing is not essential for mice in the laboratory environment, the motivation to perform the behavior may result from its reinforcing consequences. Decreased engagement in activities like burrowing may be seen as an alteration in motivation to engage in non-essential but self-rewarding activities (Cunningham et al., 2007; Teeling et al., 2007), which can be induced by various impacts on the animals' general condition and wellbeing, such as disease, pain, distress, or other impairment. Thus, the results of burrowing performance testing imply that there might be a balance between the motivation to burrow and an individuals' depression of general condition or level of pain experienced. During the animals' recovery from the noxious insult and the regeneration of wellbeing, the motivation to burrow will prevail over pain and impairment. Thus, the latency to burrow could act as an indicator of the duration of pain and reduced wellbeing in the individual. This interpretation of burrowing performance is supported by other studies hinting at an approximate time frame regarding the possible duration of pain in the laparotomy model (Arras et al., 2007; Langford et al., 2010).

In general, the prolongation of latency to burrow was in clear accordance with previous results from telemetric measurements of heart rate and heart rate variability values, as well as with decreased body weight progression and food consumption in an almost identical laparotomy model conducted in our laboratory (Arras et al., 2007). From our former study and from the findings presented here, post-operative pain is suggested as being the cause of the alterations in physiology and behavior, although symptoms of pain were not apparent upon short-term (2-10 $\mathrm{min}$ ) observation of the appearance, posture, and spontaneous locomotion of the animals. Such absence of obvious signs of pain after minor and moderate surgery in laboratory mice is commonly known from anecdotal evidence and from the experiences in the worldwide laboratory routine of embryo transfer and vasectomy, which both normally include laparotomy. Since treatment with a pain killer restored the burrowing performance to very close to the level of the control groups (anesthesia only, anesthesia + analgesia), pain seems the most likely reason for the decreased burrowing behavior.

\section{REFERENCES}

Adams, N., and Boice, R. (1981). Mouse (Mus) burrows - effects of age, strain, and domestication. Anim. Learn. Behav. 9, 140-144.

Arras, M., Rettich, A., Cinelli, P., Kasermann, H. P., and Burki, K.
(2007). Assessment of post-laparotomy pain in laboratory mice by telemetric recording of heart rate and heart rate variability. BMC Vet. Res. 3,16 .

Baumans, V. (2004). Use of animals in experimental research: an ethical

As other studies found a decrease in burrowing activity without a corresponding decrease in Open Field activity (Teeling et al., 2007), it seems unlikely that a pure mechanical impairment caused the observed prolongation of latency to burrow after surgery. While this study cannot deliver proof of pain in the laparotomy model, and the impact of anesthesia (as seen in the anesthesia only group) should not be underestimated, our results, together with cited literature observations, again support pain as the explanation for decreased burrowing behavior, while simultaneously suggesting burrowing behavior as a sensitive and easy to observe indicator of pain in laboratory mice. Since burrowing is an operant behavior, it may also be useful for testing of supraspinal drug targets, in contrast to the mainly peripheral acting analgesic we used here.

One limitation of our study is that we have analyzed systematic data only in the most commonly used C57BL/J inbred strain. However, burrowing testing has been shown to be effective in several inbred strains of laboratory mice (Solberg et al., 2006, our own preliminary data), and also works in principle also in rats, hamsters, and gerbils (Deacon, 2009), which leads us to suggest that our setting would work equally well in other mouse strains. In addition, our setup focused on the assessment of the post-operative condition of mice after laparotomy. The reproducibility of the experimental setting and the critical measuring window of 7-9 h might be proved in the future in other models of surgery or other interventions that can induce persistent pain, noxious stimuli of moderate duration, or impairment of general condition and wellbeing.

In summary, the impact of laparotomy on the general condition and wellbeing of laboratory animals was assessed using a test setting based on the natural burrowing behavior of mice. The setting was easy and convenient to perform in the animal's home cage under routine laboratory conditions. As the results of our tests allowed us to discriminate between groups of mice that were treated with a pain killer for surgery or not, burrowing performance appears to be correlated with post-operative pain, and may give hints on its duration in individual mice.

This practical setting could be tested in the future in other surgical models and in other strains of mice as a standard evaluation method for post-operative depression of general condition and wellbeing, including mild-to-moderate degrees of post-operative pain. The reproducibility of the method in other approaches and its value as a test in basic research into the mechanisms and pathophysiology of pain, and in the searching for novel pain killers is a promising avenue of research for further study.

\section{ACKNOWLEDGMENTS}

This work was sponsored by grants from the Federal veterinary office (Bern, Switzerland), and UBS foundations. The authors would like to thank Robin Schneider and the staff of the central biological laboratory for support in housing mice. We thank Professor Kurt Burki for generously providing research facilities and resources.

dilemma? Gene Ther. 11(Suppl. 1), S64-S66.

Baumans, V. (2005). Science-based assessment of animal welfare: laboratory animals. Rev. Sci. Tech. 24, 503-513.

Chapman, C. R., Casey, K. L., Dubner, R., Foley, K. M., Gracely, R. H., and
Reading, A. E. (1985). Pain measurement - an overview. Pain 22, 1-31. Cunningham, C., Campion, S., Teeling, J., Felton, L., and Perry, V.H. (2007). The sickness behaviour and CNS inflammatory mediator profile induced by systemic challenge of mice with 
synthetic double-stranded RNA (poly I:C). Brain Behav. Immun. 21, 490-502.

Deacon, R. M. (2006). Burrowing in rodents: a sensitive method for detecting behavioral dysfunction. Nat. Protoc. 1, 118-121.

Deacon, R. M. (2009). Burrowing: a sensitive behavioural assay, tested in five species of laboratory rodents. Behav. Brain Res. 200, 128-133.

Deacon, R. M., Cholerton, L. L., Talbot, K., Nair-Roberts, R. G., Sanderson, D. J., Romberg, C., Koros, E., Bornemann, K. D., and Rawlins, J. N. (2008). Age-dependent and -independent behavioral deficits in Tg2576 mice. Behav. Brain Res. 189, 126-138.

Deacon, R.M., Croucher,A., and Rawlins, J. N. (2002). Hippocampal cytotoxic lesion effects on species-typical behaviours in mice. Behav. Brain Res. 132, 203-213.

Deacon, R. M., Penny, C., and Rawlins, J. N. (2003). Effects of medial prefrontal cortex cytotoxic lesions in mice. Behav. Brain Res. 139, 139-155.

Deacon, R. M. J., Raley, J. M., Perry, V. H., and Rawlins, J.N.P. (2001). Burrowing into prion disease. Neuroreport 12, 2053-2057.

Dudek, B. C., Adams, N., Boice, R., and Abbott, M. E. (1983). Genetic influences on digging behaviors in mice (Mus musculus) in laboratory and seminatural settings. J. Comp. Psychol. 97, 249-259.
Gauthier, C., and Griffin, G. (2005). Using animals in research, testing and teaching. Rev. Sci. Tech. 24, 735-745.

Hawkins, P. (2002). Recognizing and assessing pain, suffering and distress in laboratory animals: a survey of current practice in the UK with recommendations. Lab. Anim. 36, 378-395.

Jourdan, D., Ardid, D., and Eschalier, A. (2001). Automated behavioural analysis in animal pain studies. Pharmacol. Res. 43, 103-110.

Kohn, D.F., Martin, T.E., Foley, P. L., Morris, T. H., Swindle, M. M., Vogler, G. A., and Wixson, S. K. (2007). Public statement: guidelines for the assessment and management of pain in rodents and rabbits.J. Am. Assoc. Lab. Anim. Sci. 46, 97-108.

Langford,D. J., Bailey, A. L., Chanda, M. L., Clarke, S.E., Drummond, T.E., Echols, S., Glick, S., Ingrao, J., Klassen-Ross, T., Lacroix-Fralish, M. L., Matsumiya, L., Sorge, R. E., Sotocinal, S. G., Tabaka, J. M., Wong, D., van den Maagdenberg, A. M., Ferrari, M. D., Craig, K. D., and Mogil, J. S. (2010). Coding of facial expressions of pain in the laboratory mouse. Nat. Methods 7, 447-449.

Le Bars, D., Gozariu, M., and Cadden, S. W. (2001). Animal models of nociception. Pharmacol. Rev. 53, 597-652.

Martin, T. J., Buechler, N. L., Kahn, W., Crews, J.C., and Eisenach, J. C. (2004). Effects of laparotomy on spontaneous exploratory activity and conditioned operant responding in the rat: a model for postoperative pain. Anesthesiology 101, 191-203.
Mogil, J. S. (2009). Animal models of pain: progress and challenges. Nat. Rev. Neurosci. 10, 283-294.

Mogil, J.S., and Crager, S. E. (2004). What should we be measuring in behavioral studies of chronic pain in animals? Pain 112, 12-15.

Peterson, N.C. (2004). Assessment of pain scoring. Contemp. Top. Lab. Anim. Sci. 43, 74, 76.

Roughan, J. V., Wright-Williams, S. L., and Flecknell, P.A. (2009). Automated analysis of postoperative behaviour: assessment of HomeCageScan as a novel method to rapidly identify pain and analgesic effects in mice. Lab. Anim. 43, 17-26.

Sherwin, C. M., Haug, E., Terkelsen, N., and Vadgama, M. (2004). Studies on the motivation for burrowing by laboratory mice. Appl. Anim. Behav. Sci. 88, 343-358.

Solberg, L. C., Valdar, W., Gauguier, D., Nunez, G., Taylor, A., Burnett, S., Arboledas-Hita, C., HernandezPliego, P., Davidson, S., Burns, P., Bhattacharya, S., Hough, T., Higgs, D. Klenerman,P., Cookson, W. O., Zhang, Y., Deacon, R. M., Rawlins, J. N., Mott, R., and Flint, J. (2006). A protocol for high-throughput phenotyping, suitable for quantitative trait analysis in mice. Mamm. Genome 17, 129-146.

Stasiak, K. L., Maul, D., French, E., Hellyer, P. W., and VandeWoude, S. (2003). Species-specific assessment of pain in laboratory animals. Contemp. Top. Lab. Anim. Sci. 42, 13-20.
Teeling, J. L., Felton, L. M., Deacon, R. M., Cunningham, C., Rawlins, J. N., and Perry, V. H. (2007). Sub-pyrogenic systemic inflammation impacts on brain and behavior, independent of cytokines. Brain Behav. Immun. 21 , 836-850.

van Sluyters, R. C., and Obernier, A. (2004). Guidelines for the care and use of mammals in neuroscience and behavioral research. Contemp. Top. Lab. Anim. Sci. 43, 48-52.

Conflict of Interest Statement: The authors declare that the research was conducted in the absence of any commercial or financial relationships that could be construed as a potential conflict of interest.

Received: 08 July 2010; accepted: 15 September 2010; published online: 12 October 2010.

Citation: Jirkof P, Cesarovic N, Rettich A, Nicholls F, Seifert B and Arras M (2010) Burrowing behavior as an indicator of post-laparotomy pain in mice. Front. Behav. Neurosci. 4:165. doi: 10.3389/ fnbeh.2010.00165

Copyright @ 2010 Jirkof, Cesarovic, Rettich, Nicholls, Seifert and Arras. This is an open-access article subject to an exclusive license agreement between the authors and the Frontiers Research Foundation, which permits unrestricted use, distribution, and reproduction in any medium, provided the original authors and source are credited. 